\title{
Periodontal Disease: General Aspects from Biofilm to the Immune Response Driven by Periodontal Pathogens
}

\author{
Giovanna R. Degasperi' ${ }^{1}$ Augusto Etchegaray², Larissa Marcelino², Ahron Sicard ${ }^{1}$, \\ Karina Villalpando3 ${ }^{3}$, Sérgio L. Pinheiro ${ }^{2,3}$ \\ ${ }^{1}$ Immunology League, Faculty of Medicine, Pontifical Catholic University of Campinas, Campinas, Brazil \\ ${ }^{2}$ Post-Graduation in Health Sciences, Pontifical Catholic University of Campinas, Campinas, Brazil \\ ${ }^{3}$ Dental School, Pontifical Catholic University of Campinas, Campinas, Brazil \\ Email: augusto.etchegaray@puc-campinas.edu.br
}

How to cite this paper: Degasperi, G.R., Etchegaray, A., Marcelino, L., Sicard, A., Villalpando, K. and Pinheiro, S.L. (2018) Periodontal Disease: General Aspects from Biofilm to the Immune Response Driven by Periodontal Pathogens. Advances in Microbiology, 8, 1-17.

https://doi.org/10.4236/aim.2018.81001

Received: November 19, 2017

Accepted: January 12, 2018

Published: January 16, 2018

Copyright (c) 2018 by authors and Scientific Research Publishing Inc. This work is licensed under the Creative Commons Attribution International License (CC BY 4.0).

http://creativecommons.org/licenses/by/4.0/

\begin{abstract}
Periodontal disease is of inflammatory origin and affects the dental support tissues. The initial phase is characterized by the accumulation of biofilm, which is a predisposing factor for several oral pathologies. The combination of extrinsic factors such as lack of hygiene, smoking and diet, and intrinsic factors such as genetics, and major changes in the immune response associated with immune escape mechanisms of biofilm constituents are crucial to the development and progression of the disease, which when untreated entails local irreversible consequences that can lead to systemic changes. In this brief review, we describe general aspects of periodontal disease, its risk factors and ways of preventing the disease. In particular, we discuss some of the mechanisms of immune response to periodontal pathogens, as well as the escape mechanisms of these microorganisms.
\end{abstract}

\section{Keywords}

Biofilm, Periodontal Diseases, Periodontal Pathogens, Immune Response, Immune Evasion

\section{Introduction}

Periodontal disease is the most common oral condition of human population. In general, it affects about $20 \%$ to $50 \%$ of the population worldwide, with prevalence in men, especially those who live below the poverty line and are smokers [1] [2]. 
It is characterized by a chronical inflammation that can lead to the loss of the supportive tissues of affected teeth, considering the proteolytic activity of the infecting microbes and the cascade reaction of the immune system [3].

The presence of inflammatory response alters the normal microbiota and the pathogenic microbes cohabiting within the sub-gingival biofilm will contribute to increase inflammation. Studies demonstrated that local and systemic factors of the host could have an influence on disease progression. The actual alteration of local or systemic immunological response may exert influence on the diverse clinical forms of disease.

Thus, patients with periodontitis will generate specific antibodies against bacteria that are within the biofilm. Although the role of these antibodies is not yet fully explained, they characterize adaptive humoral immune response in periodontitis. The production of these antibodies is mediated by the help of $\mathrm{T}$ lymphocytes, which are fundamental for adaptive immunity.

Considering the major histocompatibility complex, the recognition of peptide antigen by the $\mathrm{T}$ cell receptor may result in activation of these cells. $\mathrm{T}$ cell activation and differentiation may take various forms as Th1, Th2, Th17 and the more recently characterized, Th9 and Th22 subtypes. It is believed that $\mathrm{T}$ cells are involved on the homeostasis of periodontal tissues and modulation of inflammatory response [4] [5] [6]. Failures that may occur in immune response against disease establishment are due to evasive mechanisms that may be exerted by pathogenic bacteria present in the sub-gingival biofilm.

\section{Risk Factors Associated to Periodontal Disease}

Periodontal disease affects the supportive tissues of the teeth, the gingiva, periodontal ligament, cementum and alveolar bone. These changes usually occur due to persistent infection and inflammation in response to the presence of pathogens such as Porphyromonas gingivalis, Prevotella intermedia and Fusobacterium nucleatum [7]. Studies have shown that these microorganisms may be involved in the pathogenesis of a number of systemic diseases [8] [9]. The oral cavity harbors a large number of bacteria. Using molecular biology, it is now possible to accurately and rapidly identify more than 700 bacterial species that make up the oral microbiota [10]. Studies show that there is a significant variation in the microbiota according to different sites of the oral cavity. This variation can be influenced by oral diseases such as caries, infections of the dental pulp, mucositis, and periodontitis [10] [11].

The host's immune response, in addition to environmental, behavioral and genetic factors, all contribute to the risk of developing periodontal disease [12]. The presence of periodontal pathogens is necessary, but not sufficient to induce periodontitis. In the normal physiological state, where there are no risk factors, the host responds adequately trying to refrain bacterial infection. However, factors such as smoking and diabetes, for instance, in addition to the immune-system evasion capacity, given by the pathogens within sub-gingival bio- 
film, will alter the immune-inflammatory responses that may lead to tissue destruction, thus establishing periodontitis [13] [14].

Gingivitis and periodontitis are the most common forms of periodontal disease. Gingivitis is an inflammation of the gingiva without loss of alveolar bone. It is induced by the plaque biofilm and can be reverted by hygiene control. Gingivitis can lead to periodontitis, which is an inflammatory condition that will turn into loss of dental supportive tissues. It is characterized by progressive and irreversible loss of alveolar bone and, finally, loss of the teeth. Signal and symptoms include erythema, edema and bleeding, deepening of the gingival sulcus and formation of abscess (Figure 1).

Periodontitis can be classified as chronic or acute. Chronic periodontitis is more common in adults, and is generally characterized by having slow and moderate progression, while being diagnosed according to clinical signals such as an increase and deepening of the depth in periodontal probing (Figure 1), gingival inflammation and loss of alveolar bone, which can be detected by $\mathrm{x}$-ray (Figure 2) [15] [16]. Acute periodontal disease show clinical and radiographic characteristics that are similar to chronic periodontitis, however, in this case disease progression is rapid.

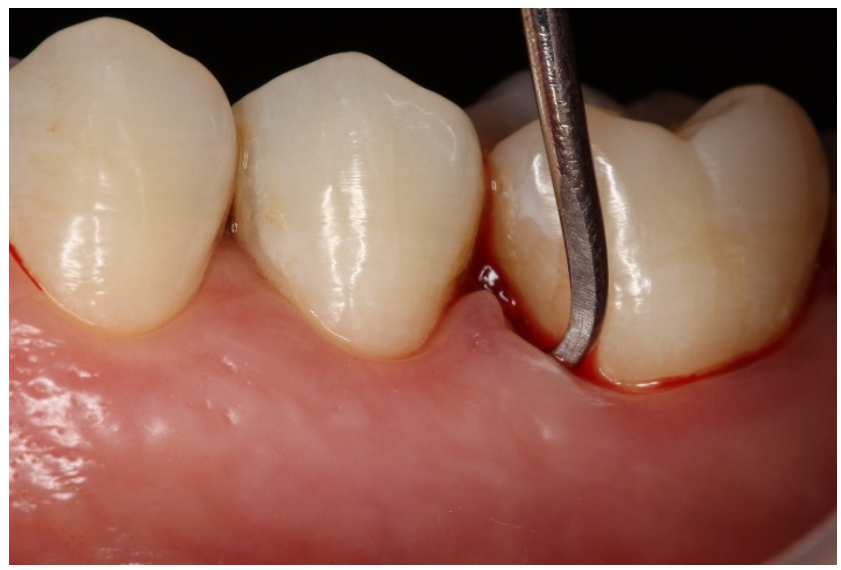

Figure 1. Picture taken at clinic showing the characteristics of periodontal disease.

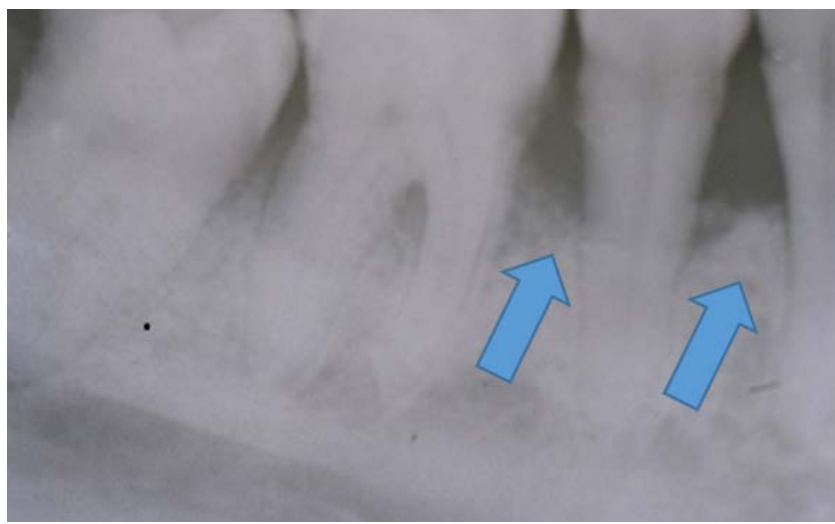

Figure 2. An example of chronic periodontitis in which the loss of alveolar bone is visible, as indicated by the arrows. 
Smoking is one of the most important risk factors in periodontal disease. A reduction in prevalence of periodontal disease is directly correlated to decreased rates of smoking [17]. Smokers present a significantly increased rate of alveolar bone loss and higher prevalence of dental loss, when compared to nonsmokers. In addition, they present unsatisfactory results through various forms of periodontal treatments [18] [19] [20] [21]. Evidence suggests that smoking induces modifications of the oral microbial flora with consequent alteration of the host's immune response [17]. In addition, nicotine has been shown to cause ruptures in periodontal tissues, either directly or indirectly through interaction with other factors [22].

Inadequate oral hygiene also contributes to the occurrence of periodontal disease, since lack of adequate brushing and other measures of oral hygiene can increase bacterial deposition and dental plaque formation on teeth and gums, thus favoring the establishment of inflammatory processes within periodontal tissues [23]. Studies have shown that there is a correlation between inadequate oral hygiene and biofilm accumulation, which is also connected to high prevalence and severity of periodontal disease [24] [25]. Additionally, inadequate oral hygiene may increase the risk factor of periodontal disease for patients with type 2 diabetes, thus contributing to disease progression [26] [27]. Type 2 diabetes is associated with destruction of the periodontal ligament, a process that can later lead to tooth loss [28] [29]. Diabetic patients that have periodontal disease will present gingival fluids and saliva that present higher concentrations of inflammatory mediators, including different types of cytokines, when compared to the non-diabetic individuals that have periodontal disease [30].

The use of certain medications and stress similarly favor periodontal disease. Vulnerability to infections and periodontal diseases intensifies when the salivary flow is reduced due to certain medications [31]. The most common medications that can reduce the salivary flux and produce a dry mouth state include tricyclic antidepressants, atropine, antihistamine and beta-blockers [32]. Reduction of salivary flow may also occur because of stress [21]. Rai et al. observed a positive association between the stress score and salivary stress markers, such as cortisol [33]. The depressed individuals showed higher concentrations of cortisol in the gingival fluid, responding in an inadequate way to the treatment for periodontal disease. Academic stress also results in inadequate oral hygiene and consequent gingiva inflammation mediated by interleukin- $1 \beta$ [21]. Another cause of mouth dryness is associated with chemotherapy and radiotherapy of the head and neck in patients with neoplasias. For instance, children in chemotherapy for leukemia treatment [34]. The severity of these treatments induce xerostomia and the problems associated to low salivary flux [35].

In addition to the risk factors discussed above, those considered as non-modifiable risks are age and hereditary factors, which also contribute to periodontal disease (Figure 3). With regard to age, the risk increases, explaining the high prevalence of periodontal disease that is observed in the aged population. 


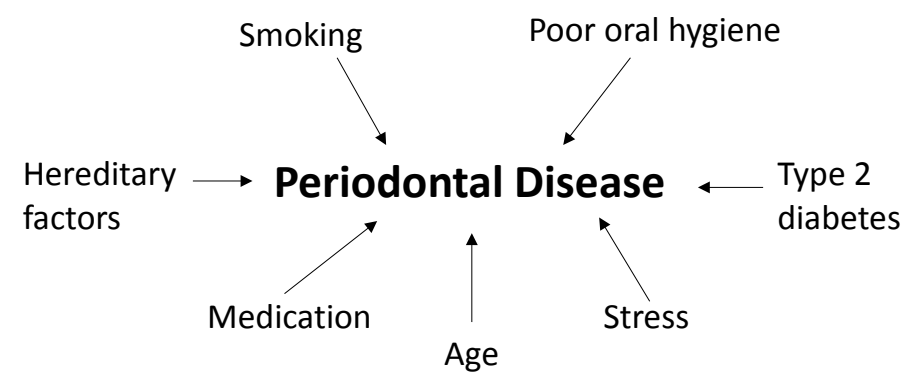

Figure 3. Risk factors that are associated to periodontal disease.

\section{Innate and Adaptive Immune Response to Oral Bacteria and the Immune Evasive Characteristics of Periodontal Pathogens}

In periodontal disease, microorganisms that are present at the sub-gingival biofilm induce the production of cytokines and chemokines by the gingival epithelium, resulting on the expression of adhesion molecules, increased permeability of the capillaries and migration of dendritic cells and neutrophils through the junction epithelium and gingival sulcus [36] [37]. The migration of neutrophils into the gingival sulcus is guided by IL-8, the chemokine produced by gingival epithelial cells [38]. In addition to the homing stimulus function or cellular trafficking, chemokines induce other biological processes, such as angiogenesis and cell proliferation, and are involved in the apoptosis of some cell types. It is also important to emphasize that the bacterial peptides themselves are also chemotactic for inflammatory cells [39] [40] [41] [42].

Phagocytes act in the capture of periodontopathic biofilm bacteria and produce pro-inflammatory cytokines that perpetuate inflammation, as well as regulate the amplitude and duration of the immune response [37]. Especially in phagocytes, the secretion of cytokines is dependent on the activation of nuclear factor NFkB after recognition of PAMPs as LPS via the TLR pathway [43]. After this type of microbial recognition, innate response pro-inflammatory cytokines that are produced in periodontal disease are, TNF- $\alpha$, IL- $1 \beta$ and IL-6 [44]. TNF- $\alpha$ regulates IL- 1 and IL- 6 production and is also correlated with extracellular matrix degradation and bone re-sorption through actions that promote RANKL secretion [45] [46] [47].

The adaptive immune response in periodontal disease is characterized by the processing and presentation of bacterial biofilm antigens, especially by dendritic cells and neutrophils that are configured with antigen-presenting cells (APCs) in this scenario [48]. T-lymphocytes that are part of the adaptive immune response recognize these antigens that are presented by the major histocompatibility complex (MHC) of these cells. In response to the recognition, T-lymphocytes produce cytokines. Classically, T-lymphocytes were classified into subsets based on the expression of $\mathrm{CD} 4^{+}$or $\mathrm{CD} 8^{+}$cell surface molecules. These molecules originate in the thymus during positive and negative $\mathrm{T}$-cell selections. $\mathrm{CD}^{+}$cells have cytotoxic activity and contribute to the progression of the inflammatory 
process in periodontal disease, but the exact mechanism of their action has not been elucidated [49]. TCD $4^{+}$cells were initially subdivided into two subsets, designated Th1 and Th2, based on their cytokine production patterns. Th1 cells secrete IL- 2 and IFN- $\gamma$, whereas Th2 cells produce IL-5, IL-6, IL-4, IL-10 and IL-13 [50]. Th1 is an important standard in the response against intracellular microorganisms and is responsible for inducing cell-mediated inflammation. By means of IFN- $\gamma$, which has a pro-inflammatory action, it leads to increased expression of toll-like receptors (TLRs), promotion of MHC class I and II antigen presentation, induction of chemokine secretion, phagocytosis, activation of $\mathrm{CD}^{+} \mathrm{T}$ cells and free radicals that act against pathogenic microorganisms and tumor cells [50]. Human and experimental data suggest that cytokine IFN- $\gamma$ is present at high levels in periodontal lesions and may contribute to the onset and progression of periodontitis by distinct mechanisms [51]. In addition, as shown in Figure 4, it has been suggested that periodontal lesions were associated with an infiltration of Th1 cells [52]. Th1 polarization is induced by cytokines such as IL-12, which is normally produced by dendritic or natural killer cells. In gingival inflammation associated with periodontal disease there is infiltration of these cells, which may be IL-12-generating cells in response to disease-associated pathogens [52].

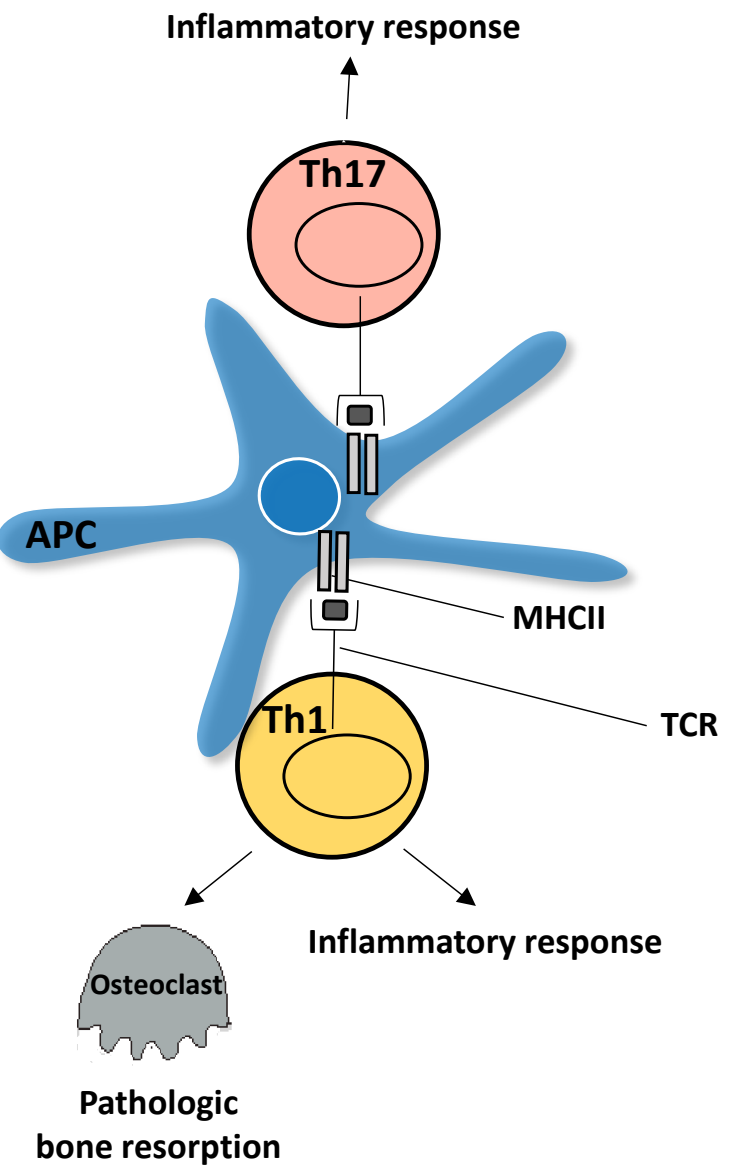

Figure 4. Immune response associated to $\mathrm{T}$ cells in periodontal disease. 
In general, $\mathrm{T}$ cells appear to play an important immune-regulatory role in periodontal disease. In a study conducted in 1985 by Yoshie et al., it was demonstrated that in athymic rats, therefore with absence of $\mathrm{CD} 4^{+}$and $\mathrm{TCD} 8^{+}$cells, the progression of periodontal disease was exacerbated. However, the immunological reconstitution with $\mathrm{TCD}^{+}$and $\mathrm{TCD} 8^{+}$thymic cells led to a reduction of the alveolar bone destruction, which is associated with periodontal disease. These results led to the conclusion that $\mathrm{T}$ lymphocytes may present important immune-regulatory functions [53].

$\mathrm{T}$ cell subsets are also important in the behavior of B cells. For example, Th1 cells stimulate the secretion of immunoglobulin G2 (IgG2), whereas Th2 cells increase the secretion of IgG1. In this way, both subtypes stimulate the humoral immune response. Some studies have shown that periodontal pathogens stimulate antibody production by B cells, most likely by $\mathrm{T}$ cells [54].

Two other well-defined subsets of $\mathrm{T}$ cells are Th17 and T regulatory (Tregs), with effector and suppressor roles respectively [55] [56]. Th17 is characterized by the unique production of IL-17 and IL-22. Th17 lymphocytes, as well as the Th1 subset, play a role in stimulating osteoclastogenesis [57]. Literature data demonstrate that Th17 cells are present in regions of inflammation that characterize periodontitis, and Th17 cytokines such as IL-17 were found in periodontal lesions (Figure 4) [58].

In vitro, antigens present on the outer membrane of $P$. gingivalis induce the production of IL-17 [59]. Furthermore, biopsy specimens of periodontal lesions from patients with chronic periodontitis, showed an increase in IL-17 mRNA [58]. IL-17, as well as the Th1-responsive IFN- $\alpha$ can stimulate RANKL expression in periodontal osteoblasts. The binding of RANKL to the osteoclast RANK receptor promotes the decrease of alveolar bone (Figure 4). The stimulation of RANKL expression is associated with several bone pathologies such as rheumatoid arthritis, bone tumors, osteoporosis, odontogenic lesions and periodontitis [60].

Treg cells secrete TGF- $\beta$ and IL-10 and, therefore, are necessary for the regulation of inflammatory responses [61]. They play a critical role in tolerogenic responses and suppression of inflammatory responses against auto-antigens. Regulatory $\mathrm{T}$ cells are $\mathrm{CD} 4^{+}$and $\mathrm{CD} 25^{+} \mathrm{T}$ cells and express the FOXP-3 transcription factor, which directs their functions. They are classified into natural (Treg) and adaptive ( $\operatorname{Tr} 1, T h 3)$ cells, with Treg being specialized in protection against auto-antigens and exacerbated inflammatory processes [62]. It has been observed that they can be found in sites of periodontal disease, exerting a protective role of tissue damage induced by the disease [56] [63] [64]. Ernst et al. have also demonstrated that Treg cells reduce RANKL expression in periodontal osteoblasts [65].

\section{Biofilm and Evasion Mechanisms against Immune Cells in Periodontal Disease}

It was estimated that over 700 species of bacteria can colonize specific sites in the 
oral cavity accordingly with tropisms resulting in three-dimensional structure and organized biofilms [66]. Biofilms have been shown to have high persistence, since infections are established and innate and adaptive immune responses are simultaneously activated and frequently, as in periodontitis, these responses are the bases for collateral tissue damage [67]. In addition, there are reports on the literature showing that bacteria present in the biofilm are more resistant to phagocytic activity of neutrophils and that their replication capacity is higher in this environment [68].

Thus, the oral microbiome exists essentially within biofilms along with the various niches in the oral cavity. These complexes form ecosystems that directly contribute to maintain health when the system is in balance. However, specific ecological changes in this the commensal microbiome allows the overcome of pathogens, allowing their pathogenic potential to cause disease. The oral microbial ecology is influenced by numerous characteristics, including temperature, $\mathrm{pH}$, atmosphere, endogenous nutrients and a variety of surfaces for the establishment of biofilms [69].

Bacterial adherence to mucosal and tooth surfaces, as well as bacterial co-aggregation, are essential steps for colonization of various oral bacterial species. In the case of $P$. gingivalis, for example, the development of major fimbriae are critical determinants for each of these processes, which are the key adhesive determinants on the cell surface of this pathogen. Fimbriae from $P$. gingivalis attach to a variety of host components, including epitelial cells, fibroblasts, human salivary macromolecules and, within experimental systems, it has been shown that these are critical for successful colonization [70]. With regard to co-aggregation, it was identified that gingipains are components induced by $P$. gingivalis that significantly alter the co-aggregation of Actinobacillus actinomycetemcomitans, influencing biofilms that are formed by this oral pathogen [71]. These types of findings indicate that the multiple surface components of these oral bacteria act in a cooperative manner to enable specific co-aggregation and with specific properties that allow the formation of complex biofilm structures.

As a characteristic, the bacteria present in the biofilm have immune system evasion mechanisms. $P$. gingivalis is a proteolytic microbe and thus produces proteases that are capable to degraded IL- 8 and MCP-1, which are produced by endothelial cells and are important to maintain periodontal health [72]. They are also able to block the production of immunoglobulins and to attack and degrade complement components such as $\mathrm{C} 3 \mathrm{a}$ and $\mathrm{C} 3 \mathrm{~b}$. C3b is a complement system protein that is important for the opsonization of microorganisms, as it facilitates their phagocytosis. It is also an important component of the C5 convertase, an enzymatic complex essential for the synthesis of the C5a component. C5a and $\mathrm{C} 3 \mathrm{a}$ are known as anaphylatoxins and they thrive on the phagocytic chemotaxis, since these cells have receptors for $\mathrm{C} 5 \mathrm{a}$ and $\mathrm{C} 3 \mathrm{a}$. Therefore, the proteolytic capacity of the evasive microbes, which act on the breakdown of the $\mathrm{C} 3 \mathrm{~b}$ protein, will block opsonization, thus reducing phagocytosis. Thus, the destruction of 
these two components of the complementary system by the action of proteases facilitates the survival of invasive bacteria [73]. Some bacteria will also use surface molecules to escape the action of the complement system, as in the case of HRgpA gingipain from $P$. gingivalis [74].

Additional microbes are present in the biofilm, which strategically have the ability to prevent immune response. An example is that of $P$. intermedia, which produces nucleases capable of degrading neutrophil extracellular traps (NETs), therefore limiting the neutrophil-protective functions [75]. The targets of the evasive mechanism of periodontopathic bacteria are summarized in Figure 5.

\section{Prevention of Periodontal Disease}

The World Health Organization recommends employing integrated public health measures with preventive strategies that should be based on common risk and multifactorial approaches. Risk factors such as smoking, stress and low socioeconomic status are associated with periodontal disease as well as other chronic systemic diseases. Therefore, inclusion of strategies to prevent oral diseases and chronic systemic diseases can reduce the level of periodontal disease in the population [69]. Good oral hygiene practices such as regular tooth brushing and flossing are effective in preventing periodontitis. However, despite the great significance of dental brushing, about half of the population brush only twice a day [70].

The use of fluoride, chlorhexidine, triclosan, essential oils and zinc in toothpastes, mouthwashes and gels associated with brushing all contribute to prevent disease development [71] [72]. He et al. conducted a double-blind randomized trial to investigate the antimicrobial role of fluoride dentifrice in periodontal disease and found a significant reduction in gingival bleeding over a period of 2 months [73].

Diet is also an important factor for disease prevention [74]. Nishida et al. used a sample of 12,419 adults and showed that there was an increased risk of periodontal disease due to poor dietary intake of vitamin C. The authors also observed

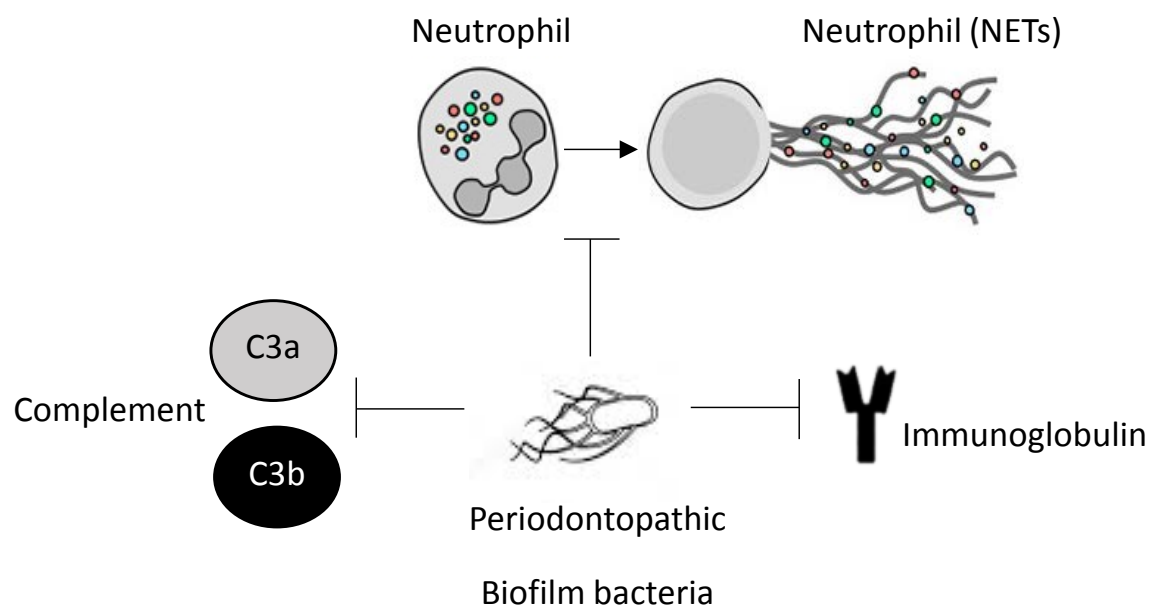

Figure 5. Evasion mechanisms against immune response in periodontal disease. 
a dose-response relationship between vitamin $C$ and disease severity [75]. Vitamin $\mathrm{C}$ has antioxidant properties that help to reduce the production of reactive oxygen radicals formed during the inflammatory process [74]. Furthermore, a diet that is rich in fruits, vegetables and low fat and sugars contributes to maintain a healthy periodontal tissue, and low calorie intake reduce inflammatory changes, lowering the chances of tissue damage in periodontal disease [75].

\section{Treatment and Future Perspectives}

Although the non-surgical and surgical periodontal treatments have been used for many years, they still remain the basis of periodontal therapy. Novel technological approaches have increased the options for therapies, including novel periodontal instrumentation. However, it is now recognized that non-surgical and surgical periodontal therapy must be based on the control of bacterial biofilm [76].

The development of ultrasonic and sonic instruments with thin-tipped models has contributed to reduce damages due to the use of instruments, considering the improvements in accessibility, which cause less damage to the root surface [77]. In parallel, some studies have demonstrated that similarly clinical and microbiological results can be obtained using manual instrumentation in comparison with treatments that use sonic and ultrasonic instruments [78].

The use of antiseptics such as povidone-iodine and chlorhexidine gluconate to irrigate the periodontal pocket has also been tested. These chemicals are used in non-surgical periodontal treatment after mechanical instrumentation, in order to suppress dental biofilm. Studies have shown a reduction in periodontal pathogens and depth of probing when compared to scaling and root planing alone [79]. However, the effec of antiseptics is only transient and of short-term as the gingival crevicular fluid is replaced within the pocket at short intervals (every 90 seconds) [76].

Similarly, the release of antimicrobial agents into the periodontal pocket has been investigated after scaling and root straightening by using slow release systems. Several antimicrobials and various drug delivery systems have been tested in order to maintain high antimicrobial levels in the crevicular fluid and, on the other hand, which have minimal systemic absorption. The most commonly tested antimicrobials were tetracycline, minocycline, metronidazole and chlorhexidine. The results showed modest and short-term improvement in depth of probing reduction, when compared to scaling and root planing alone [80].

On the other hand, the use of systemic antimicrobial adjunctive treatment to non-surgical periodontal therapy has shown benefits for patients with aggressive periodontitis and advanced periodontitis, including those with the presence of deep periodontal pockets, thus reducing the need for additional therapy. The combination of amoxicillin $500 \mathrm{mg}$ and metronidazole $400 \mathrm{mg}$ for 7 to 14 days is the best-documented antimicrobial regimen [76].

Despite the benefits of the use of systemic antimicrobials, the risk of adverse 
effects, including the development of bacterial resistance, the interactions with other drugs and possible allergic reactions must be considered [81].

More recently, the advent of antimicrobial photodynamic therapy (PDT) has also made substantial contributions. PDT involves the use of low-power lasers of appropriate wavelength to reduce microorganisms by a light-activated photosensitizer. This therapy can be used alone or in conjunction with root scaling and straightening [76]. Adjunctive PDT used with root scaling and straightening has shown short-term benefits, while microbiological results are contradictory [82]. Long-term randomized controlled trials, supported by the presentation of clinical and microbiological data, besides a detailed cost and benefit analysis, are required to support the efficacy of this therapy [76]. Finally, the use of stem cells for periodontal regeneration and the applications of antibody therapies may be promising alternatives in the treatment of periodontal disease [83].

\section{Conclusions}

Periodontal disease is an immune-inflammatory condition caused by a polymicrobial structure, described as the dental biofilm. Several risk factors such as smoking, poor oral hygiene, diabetes, medication, age, hereditary origins, and stress are related to periodontal diseases. In addition to them are the numerous bacterial species formed in the dental biofilm, and the specific pathogen that is responsible for the initiation or progression of the disease cannot be distinguished, although the anaerobic Gram-negative species are in fact associated with advanced forms of the disease. Negligence in oral hygiene may result in maturation of the biofilm and thus trigger host response, which is clinically taken as periodontitis. During this inflammatory condition, an immune reaction is triggered against periodontopathic biofilm bacteria. This response involves elements of the immune response and adaptive immune system. However, some microorganisms developed strategies to escape protective immunity, often by manipulating key components of the immune response through proteolysis: a mechanism that creates specific conditions for their survival at sub-gingival pockets.

The understanding of the behavior of the microorganisms of the dental biofilm against the action of the immune system in the periodontal disease helps in the establishment of new forms of treatment and prevention of the disease. Effective and established utilization of the knowledge of immunological processes occurring in the periodontium is found in modern treatment protocols. For example, $P$. gingivalis has been a target for monoclonal antibody therapy. Specific monoclonal IgG antibodies were found to be effective in improving the quality of the immune response in experimental model to periodontal disease. Also, against inflammatory processes, lipoxins and resolvins are new drugs proposed that act as lipid inflammatory suppressors. In this context, studies show the efficacy of resolvins in suppression of neutrophil infiltration, in inflammation and better periodontal regeneration processes. Modulating the complement 
system also seems to be a comprehensive strategy for treatment of the disease. It has been shown that the PM5-C5a receptor antagonist PMX-53 blocked periodontal inflammation and bone loss in a periodontitis model induced by $P$. gingivalis, regardless of whether it had been administered before or after the onset of the disease.

The best chance of clinical improvement seems to be associated with the implementation of complementary adjunctive treatment strategies (mechanical, chemical and alternative) leading to the periodontal equilibrium. Modulation of the host's immune response, in combination with the conventional treatment, directs a curative response for the disease. Immune-modulatory therapies can be a fundamental part of future research associated with the treatment of the disease, with the example of therapeutic antibodies such as infliximab that helps to control exacerbated inflammation, and may represent the future of adjunctive periodontal therapy, thus reducing the use of antimicrobials. Therefore, considering the outcome of periodontal disease, it is advisable to establish preventive measures in order to avoid the incidence of the disease, as it reflects on the quality of life of the affected population and generates additional expenses to the patient and to the health system.

\section{Acknowledgements}

L. M. received a scholarship from CAPES, Brazil.

\section{References}

[1] Kholy, K.E., Genco, R.J. and Van Dyke, T.E. (2015) Oral Infections and Cardiovascular Disease. Trends in Endocrinology \& Metabolism, 26, 315-321. https://doi.org/10.1016/j.tem.2015.03.001

[2] National Center for Health Statistics. Health, the United States, 2014: With Special Feature on Adults Aged 55-64. Hyattsville, MD. 2015.

[3] Bartold, P.M. and Van Dyke, T.E. (2013) Periodontitis: A Host-Mediated Disruption of Microbial Homeostasis. Unlearning Learned Concepts. Periodontology 2000, 62, 203-217.

[4] Gemell, E., Yamazak, K. and Gregory, J. (2007) The Role of T Cells in Periodontal Disease: Homeostasis and Autoimmunity. Periodontology 2000, 43, 14-40. https://doi.org/10.1111/j.1600-0757.2006.00173.x

[5] Azuma, M. (2006) Fundamental Mechanisms of Host Immune Responses to Infection. Journal of Periodontal Research, 41, 361-373. https://doi.org/10.1111/j.1600-0765.2006.00896.x

[6] Teng, Y.T., et al. (2000) Functional Human T-Cell Immunity and Osteoprotegerin Ligand Control Alveolar Bone Destruction in Periodontal Infection. The Journal of Clinical Investigation, 106, 59-67. https://doi.org/10.1172/JCI10763

[7] Kononen, E. and Muller, H.P. (2014) Microbiology of Aggressive Periodontitis. Periodontology 2000, 65, 46-78. https://doi.org/10.1111/prd.12016

[8] Kozarov, E.V., Dorn, B.R., Shelburne, C.E., et al. (2005) Human Atherosclerotic Plaque Contains Viable Invasive Actinobacillus actinomycetemcomitans and Porphyromonas gingivalis. Arteriosclerosis, Thrombosis, and Vascular Biology, 25, 
17-18. https://doi.org/10.1161/01.ATV.0000155018.67835.1a

[9] Venkataramani, A., Santo-Domingo, N.E. and Main, D.M. (1994) Actinobacillus Actinomycetemcomitans Pneumonia with Possible Septic Embolization. Chest, 105, 645-646.

[10] Dewhirst, F.E., Chen, T., Izard, J., et al. (2010) The Human oral Microbiome. Journal of Bacteriology, 192, 5002-5017.

[11] Aas, J.A., et al. (2005) Defining the Normal Bacterial Flora of the Oral Cavity. Journal of Clinical Microbiology, 43, 5721-5732. https://doi.org/10.1128/JCM.43.11.5721-5732.2005

[12] Yucel-Lindberg, T. and Bage, T. (2013) Inflammatory Mediators in the Pathogenesis of Periodontitis. Expert Reviews in Molecular Medicine, 15, 1-22.

[13] D’Aiuto, F., et al. (2017) Evidence Summary: The Relationship between Oral Diseases and Diabetes. British Dental Journal, 222, 944-948.

[14] Alexandridi, F., Tsantila, S. and Pepelassi, E. (2017) Smoking Cessation and Response to Periodontal Treatment. Australian Dental Journal. https://doi.org/10.1111/adj.12568

[15] American Academy of Periodontology (2000) Parameter on Chronic Periodontitis with Slight to Moderate Loss of Periodontal Support. Periodontology 2000, 71, 853-855.

[16] Armitage, G.C. (1999) Development of a Classification System for Periodontal Diseases and Conditions. Annals of Periodontology, 4, 1-6. https://doi.org/10.1902/annals.1999.4.1.1

[17] Bergstrom, J. (2014) Smoking Rate and Periodontal Disease Prevalence: 40-Year Trends in Sweden 1970-2010. Journal of Clinical Periodontology, 41, 952-957. https://doi.org/10.1111/jcpe.12293

[18] Sanz, M., D’Aiuto, F., Deanfield, J. and Fernandez-Avilés, F. (2010) European Workshop in Periodontal Health and Cardiovascular Disease-Scientific Evidence on the Association between Periodontal and Cardiovascular Diseases: A Review of the Literature. European Heart Journal Supplements, 12, 3-12.

https://doi.org/10.1093/eurheartj/suq003

[19] Underner, M., Maes, I., Urban, T. and Meurice, J.C. (2009) Effects of Smoking on Periodontal Disease. Revue des Maladies Respiratoires, 26, 1057-1073. https://doi.org/10.1016/S0761-8425(09)73533-8

[20] Albandar, J.M., Streckfus, C.F., Adesanya, M.R. and Winn, D.M. (2000) Cigar, Pipe, and cigarette Smoking as Risk Factors for Periodontal Disease and tooth Loss. Journal of Periodontology, 71, 1874-1881. https://doi.org/10.1902/jop.2000.71.12.1874

[21] Reners, M. and Brecx, M. (2007) Stress and Periodontal Disease. International Journal of Dental Hygiene, 5, 199-204. https://doi.org/10.1111/j.1601-5037.2007.00267.x

[22] Nociti, F.H., et al. (2001) Histometric Evaluation of the Effect of Nicotine Administration on Periodontal Breakdown: An In Vivo Study. Journal of Periodontal Research, 36, 361-366. https://doi.org/10.1034/j.1600-0765.2001.360603.x

[23] Oliveira, C., Watt, R. and Hamer, M. (2010) Toothbrushing, Inflammation, and Risk of Cardiovascular Disease: Results from Scottish Health Survey. BMJ, 340, 1-6. https://doi.org/10.1136/bmj.c2451

[24] Albandar, J.M. (2002) Global Risk Factors and Risk Indicators for Periodontal Diseases. Periodontology, 29, 177-206. 
https://doi.org/10.1034/j.1600-0757.2002.290109.x

[25] Axelsson, P., Lindhe, J. and Nyström, B. (1991) On the Prevention of Caries and Periodontal Disease. Results of a 15-Year Longitudinal Study in Adults. Journal of Clinical Periodontology, 18, 182-189. https://doi.org/10.1111/j.1600-051X.1991.tb01131.x

[26] Casanova, L., Hughes, F.J. and Preshaw, P.M. (2014) Diabetes and Periodontal Disease: A Two-Way Relationship. British Dental Journal, 217, 433-437. https://doi.org/10.1038/sj.bdj.2014.907

[27] Preshaw, P.M. and Bissett, S.M. (2013) Periodontitis: Oral Complication of Diabetes. Endocrinology and Metabolism Clinics of North America, 42, 849-867. https://doi.org/10.1016/j.ecl.2013.05.012

[28] Grossi, S.G., et al. (1994) Assessment of Risk for Periodontal Disease. I. Risk Indicators for Attachment Loss. Journal of Periodontology, 65, 260-267. https://doi.org/10.1902/jop.1994.65.3.260

[29] Patel, M.H., Kumar, J.V. and Moss, M.E. (2013) Diabetes and Tooth Loss: An Analysis of Data from the National Health and Nutrition Examination Survey, 2003-2004. The Journal of the American Dental Association, 144, 478-485. https://doi.org/10.14219/jada.archive.2013.0149

[30] Chapple, I.L. and Genco, R. (2013) Working Group 2 of the Joint EFP/AAP Workshop. Diabetes and Periodontal Diseases: Consensus Report of the Joint EFP/AAP Workshop on Periodontitis and Systemic Diseases. Journal of Periodontology, 84, 106-112. https://doi.org/10.1902/jop.2013.1340011

[31] Güncü, G.N., Tözüm, T.F. and Caglayan, F. (2005) Effects of Endogenous Sex Hormones on the Periodontium-Review of Literature. Australian Dental Journal, 50, 138-145. https://doi.org/10.1111/j.1834-7819.2005.tb00352.x

[32] Scully, C. (2003) Drug Effects on Salivary Glands: Dry Mouth. Oral Diseases, 9, 165-176. https://doi.org/10.1034/j.1601-0825.2003.03967.x

[33] Rai, B., Kaur, J., Anand, S.C. and Jacobs, R. (2011) Salivary Stress Markers, Stress, and Periodontitis: A Pilot Study. Journal of Periodontology, 82, 287-292. https://doi.org/10.1902/jop.2010.100319

[34] Padmini, C. and Bai, K.Y. (2014) Oral and Dental Considerations in Pediatric Leukemic Patient. ISRN Hematology, 2014, Article ID: 895721.

[35] Barbería, E., Hernandez, C., Miralles, V. and Maroto, M. (2008) Paediatric Patients Receiving Oncology Therapy: Review of the Literature and Oral Management Guidelines. European Journal of Paediatric Dentistry, 9, 188-194.

[36] Takeichi, O., et al. (2000) Cytokine Profiles of T-Lymphocytes from Gingival Tissues with Pathological Pocketing. Journal of Dental Research, 79, 1548-1555. https://doi.org/10.1177/00220345000790080401

[37] Amano, A. (2010) Bacterial Adhesins to Host Components in Periodontitis. Periodontology, 52, 12-37. https://doi.org/10.1111/j.1600-0757.2009.00307.x

[38] Tonetti, M.S., et al. (1994) Localized Expression of mRNA for Phagocyte-Specific Chemotactic Cytokines in Human Periodontal Infections. Infection and Immunity, 62, 4005-4014.

[39] Rossi, D. and Zlotnik, A. (2000) The Biology of Chemokines and Their Receptors. Annual Review of Immunology, 18, 217-242. https://doi.org/10.1146/annurev.immunol.18.1.217

[40] Zlotnik, A. and Yoshie, O. (2000) Chemokines: A New Classification System and Their Role in Immunity. Immunity, 12, 121-127. 
https://doi.org/10.1016/S1074-7613(00)80165-X

[41] Ridiandries, A., Tan, J.T. and Bursill, C.A. (2016) The Role of CC-Chemokines in the Regulation of Angiogenesis. International Journal of Molecular Sciences, 17, 2-16. https://doi.org/10.3390/ijms17111856

[42] Fogarty, C.E. and Bergmann, A. (2015) The Sound of Silence: Signaling by Apoptotic Cells. Current Topics in Developmental Biology, 114, 241-265. https://doi.org/10.1016/bs.ctdb.2015.07.013

[43] Mayadas, T.N., Cullere, X. and Lowell, C.A. (2014) The Multifaceted Functions of Neutrophils. Annual Review of Pathology, 9, 181-218. https://doi.org/10.1146/annurev-pathol-020712-164023

[44] Gomes, F.I., et al. (2016) Inflammatory Cytokines Interleukin-1 $\beta$ and Tumour Necrosis Factor- $\alpha$-Novel Biomarkers for the Detection of Periodontal Diseases: A Literature Review. Journal of Oral \& Maxillofacial Research, 7,

[45] Graves, D. (2008) Cytokines That Promote Periodontal Tissue Destruction. Journal of Periodontology, 79, 1585-1591. https://doi.org/10.1902/jop.2008.080183

[46] Graves, D.T., Oates, T. and Garlet, G.P. (2011) Review of Osteoimmunology and the Host Response in Endodontic and Periodontal Lesions. Journal of Oral Microbiology, 17, https://doi.org/10.3402/jom.v3i0.5304

[47] Chen, B., et al. (2014) RANKL Expression in Periodontal Disease: Where Does RANKL Come from? BioMed Research International, 1-7. https://doi.org/10.1155/2014/731039

[48] Song, L., Dong, G., Guo, L. and Graves, D.T. (2017) The Function of Dendritic Cells in Modulating the Host Response. Molecular Oral Microbiology. https://doi.org/10.1111/omi.12195

[49] Cifcibasi, E., et al. (2015) The Role of Activated Cytotoxic T Cells in Etiopathogenesis of Periodontal Disease: Does It Harm or Does It Heal? Scientific Reports, 5.

[50] Raphael, I., Nalawade, S., Eagar, T.N. and Forsthuber, T.G. (2015) T Cell Subsets and Their Signature Cytokines in Autoimmune and Inflammatory Diseases. Cytokine, 74, 5-17. https://doi.org/10.1016/j.cyto.2014.09.011

[51] Dutzan, N., et al. (2009) Levels of Interferon-Gamma and Transcription Factor T-Bet in Progressive Periodontal Lesions in Patients with Chronic Periodontitis. Journal of Periodontology, 80, 290-296. https://doi.org/10.1902/jop.2009.080287

[52] Jotwani, R., et al. (2001) Mature Dendritic Cells Infiltrate the T-Cell Rich Region of Oral Mucosa in Chronic Periodontitis: In Situ, In Vivo and In Vitro Studies. The Journal of Immunology, 167, 4693-700. https://doi.org/10.4049/jimmunol.167.8.4693

[53] Yoshie, H., et al. (1985) Periodontal Bone Loss and Immune Characteristics of Congenitally Athymic and Thymus Cell-Reconstituted Athymic Rats. Infection and Immunity, 50, 403-408.

[54] Campbell, L., Millhouse, E., Malcolm, J. and Culshaw, S. (2016) T Cells, Teeth and Tissue Destruction What Do T Cells Do in Periodontal Disease? Molecular Oral Microbiology, 31, 445-456. https://doi.org/10.1111/omi.12144

[55] Appay, V., van Lier, R.A., Sallusto, F. and Roederer, M. (2008) Phenotype and Function of Human T Lymphocyte Subsets: Consensus and Issues. Cytometry $A$, 73, 975-983. https://doi.org/10.1002/cyto.a.20643

[56] Sallusto, F. and Lanzavecchia, A. (2009) Human Th17 Cells in Infection and Autoimmunity. Microbes and Infection, 11, 620-624.

https://doi.org/10.1016/j.micinf.2009.04.004 
[57] Gálvez, J. (2014) Role of Th17 Cells in the Pathogenesis of Human IBD. ISRN Inflammation, 1-14. https://doi.org/10.1155/2014/928461

[58] Vernal, R., Dutzan, N., Chaparro, A., Puente, J., Antonieta Valenzuela, M. and Gamonal, J. (2005) Levels of Interleukin-17 in Gingival Crevicular Fluid and in Supernatants of Cellular Cultures of Gingival Tissue from Patients with Chronic Periodontitis. Journal of Clinical Periodontology, 32, 383-389. https://doi.org/10.1111/j.1600-051X.2005.00684.x

[59] Oda, T., Yoshie, H. and Yamazaki, K. (2003) Porphyromonas gingivalis Antigen Preferentially Stimulates T Cells to Express IL-17 but Not Receptor Activator of NF- $\mathrm{B}$ Ligand In Vitro. Oral Microbiology and Immunology, 18, 30-36. https://doi.org/10.1034/j.1399-302X.2003.180105.x

[60] Crotti, T., Smith, M.D., Hirsch, R., et al. (2003) Receptor Activator NF kappaB ligand (RANKL) and Osteoprotegerin (OPG) Protein Expression in Periodontitis. Journal of Periodontal Research, 38, 380-387. https://doi.org/10.1034/j.1600-0765.2003.00615.x

[61] Togashi, Y. and Nishikawa, H. (2017) Regulatory T Cells: Molecular and Cellular Basis for Immunoregulation. Current Topics in Microbiology and Immunology. https://doi.org/10.1007/82_2017_58

[62] Garlet, G.P., Sfeir, C.S. and Little, S.R. (2014) Restoring Host-Microbe Homeostasis via Selective Chemoattraction of Tregs. Journal of Dental Research, 93, 834-839. https://doi.org/10.1177/0022034514544300

[63] Nakajima, T., et al. (2005) Regulatory T-Cells Infiltrate Periodontal Disease Tissues. Journal of Dental Research, 84, 639-643. https://doi.org/10.1177/154405910508400711

[64] Cardoso, C.R., et al. (2008) Characterization of CD4 (+) CD25 (+) Natural Regulatory T Cells in the Inflammatory Infiltrate of Human Chronic Periodontitis. Journal of Leukocyte Biology, 84, 311-318. https://doi.org/10.1189/jlb.0108014

[65] Ernst, C.W., et al. (2007) Diminished Forkhead Box P3/CD25 Double Positive T Regulatory Cells Are Associated with the Increased Nuclear Factor- $\mathrm{kB}$ Ligand $(\mathrm{RANKL}+) \mathrm{T}$ Cells in the Bone Resorption Lesion of Periodontal Disease. Clinical \& Experimental Immunology, 148, 271-280. https://doi.org/10.1111/j.1365-2249.2006.03318.x

[66] Zijnge, V., et al. (2012) Subgingival Biofilm Structure. Frontiers of Oral Biology, 15, $1-16$.

[67] Zelkha, S.A., Freilich, R.W., Amar, S. (2010) Periodontal Innate Immune Mechanisms Relevant to Atherosclerosis and Obesity. Journal of Periodontology, 54, 207-221. https://doi.org/10.1111/j.1600-0757.2010.00358.x

[68] Carvalho, R.P., et al. (2009) Relationship of Neutrophil Phagocytosis and Oxidative Burst with the Subgingival Microbiota of Generalized Aggressive Periodontitis. Oral Microbiology and Immunology, 24, 124-132. https://doi.org/10.1111/j.1399-302X.2008.00484.x

[69] Marsh, P.D. and Zaura, E. (2017) Dental Biofilm: Ecological Interactions in Health and Disease. Journal of Clinical Periodontology, 18, 12-22. https://doi.org/10.1111/jcpe.12679

[70] Baek, K.J., Ji, S., Kim, Y.C. and Choi, Y. (2015) Association of the Invasion Ability of Porphyromonas gingivalis with the Severity of Periodontitis. Virulence, 6, 274-281. https://doi.org/10.1080/21505594.2014.1000764

[71] Bao, K., Belibasakis, G.N., Thurnheer, T., Aduse-Opoku, J., Curtis, M.A. and Bos- 
tanci, N. (2014) Role of Porphyromonas gingivalis Gingipains in Multi-Species Biofilm Formation. BMC Microbiology, 14, 258. https://doi.org/10.1186/s12866-014-0258-7

[72] Kobayashi-Sakamoto, M., Isogai, E. and Hirose, K. (2003) Porphyromonas gingivalis Modulates the Production of Interleukin 8 and Monocyte Chemotactic Protein 1 in Human Vascular Endothelial Cells. Current Microbiology, 46, 109-114. https://doi.org/10.1007/s00284-002-3782-x

[73] Slaney, J.M. and Curtis, M.A. (2008) Mechanisms of Evasion of Complement by Porphyromonas gingivalis. Frontiers in Bioscience, 13, 188-196. https://doi.org/10.2741/2669

[74] Hajishengallis, G, Maekawa, T., Abe, T., Hajishengallis, E. and Lambris, J.D. (2015) Complement Involvement in Periodontitis: Molecular Mechanisms and Rational Therapeutic Approaches. Advances in Experimental Medicine and Biology, 865, 57-74. https://doi.org/10.1007/978-3-319-18603-0_4

[75] Doke, M., Fukamachi, H., Morisaki, H., Arimoto, T., Kataoka, H. and Kuwata, H. (2017) Nucleases from Prevotella intermedia Can Degrade Neutrophil Extracellular Traps. Molecular Oral Microbiology, 32, 288-300. https://doi.org/10.1111/omi.12171

[76] Heitz-Mayfield, L.J.A. and Lang, N.P. (2013) Surgical and Nonsurgical Periodontal Therapy. Learned and Unlearned Concepts. Journal of Periodontology, 62, 218-230. https://doi.org/10.1111/prd.12008

[77] Braun, A., Jepsen, S. and Krause, F. (2007) Subjective Intensity of Pain during U1trasonic Supragingival Calculus Removal. Journal of Clinical Periodontology, 34, 668-672. https://doi.org/10.1111/j.1600-051X.2007.01100.x

[78] Tunkel, J., Heinecke, A. and Flemmig, T.F. (2002) A Systematic Review of Efficacy of Machine-Driven and Manual Subgingival Debridement in the Treatment of Chronic Periodontitis. Journal of Clinical Periodontology, 29, 72-81, Discussion 90-71. https://doi.org/10.1034/j.1600-051X.29.s3.4.x

[79] Ribeiro, E. del P., Bittencourt, S., Sallum, E.A., Sallum, A.W., Nociti Jr, F.H. and Casati, M.Z. (2010) Non-Surgical Instrumentation Associated with Povidone-Iodine in the Treatment of Interproximal Furcation Involvements. Journal of Applied Oral Science, 18, 599-606. https://doi.org/10.1590/S1678-77572010000600011

[80] Bonito, A.J., Lux, L. and Lohr, K.N. (2005) Impact of Local Adjuncts to Scaling and Root Planing in Periodontal Disease Therapy: A Systematic Review. Journal of Periodontology, 76, 1227-1236. https://doi.org/10.1902/jop.2005.76.8.1227

[81] Serrano, C., Torres, N., Valdivieso, C., Castano, C., Barrera, M. and Cabrales, A. (2009) Antibiotic Resistance of Periodontal Pathogens Obtained from Frequent Antibiotic Users. Acta Odontológica Latinoamericana, 22, 99-104.

[82] Sgolastra, F., Petrucci, A., Gatto, R., Marzo, G. and Monaco, A. (2013) Photodynamic Therapy in the Treatment of Chronic Periodontitis: A Systematic Review and Meta-Analysis. Lasers in Medical Science, 28, 669-682.

https://doi.org/10.1007/s10103-011-1002-2

[83] Bansal, R. and Jain, A. (2015) Current Overview on Dental Stem Cells Applications in Regenerative Dentistry. Journal of Natural Science, Biology, and Medicine, 6, 29-34. https://doi.org/10.4103/0976-9668.149074 